\title{
Key Genes Associated with Tumor-Infiltrating Non-regulatory CD4- and CD8-Positive T Cells in Microenvironment of Hepatocellular Carcinoma
}

\author{
Zijun Zhao ${ }^{1} \cdot$ Chaonan Wang ${ }^{2} \cdot$ Peishan $\mathrm{Chu}^{3} \cdot \mathrm{Xin}^{\mathrm{Lu}}{ }^{1} \oplus$
}

Received: 13 April 2021 / Accepted: 6 December 2021 / Published online: 29 January 2022

(c) The Author(s) 2022

\begin{abstract}
The immune microenvironment in hepatocellular carcinoma (HCC), especially T-cell infiltration, plays a key role in the prognosis and drug sensitivity of HCC. Our study aimed to analyze genes related to non-regulatory $\mathrm{CD}^{+}$and $\mathrm{CD}^{+} \mathrm{T}$ cell in HCC. Data of HCC samples were downloaded from The Cancer Genome Atlas (TCGA) database. According to stromal and immune score retrieved by Estimation of Stromal and Immune cells in Malignant Tumor tissues using Expression data (ESTIMATE) algorithm, differentiated expressed genes (DEGs) between high and low stromal/immune scoring groups were collected. Using Cibersort algorithm, abundance of immune cells was calculated and genes related with $\mathrm{CD}^{+}$and $\mathrm{CD} 8^{+}$ $\mathrm{T}$ cells were selected. Protein-protein interaction (PPI) networks and networks of microRNA (miRNA)-target gene interactions were illustrated, in which $\mathrm{CD}^{+}$ and $\mathrm{CD}^{+} \mathrm{T}$ cell-related core genes were selected. Finally, Cox regression test and Kaplan-Meier (K-M) survival analysis were conducted. Totally, 1579 DEGs were identified, where 103 genes and 407 genes related with $\mathrm{CD}^{+}$and $\mathrm{CD} 8^{+} \mathrm{T}$ cell were selected, respectively. Each of 30 core genes related to $\mathrm{CD} 4^{+} \mathrm{T}$ cells and $\mathrm{CD} 8^{+} \mathrm{T}$ cells were selected by PPI network. Four genes each related with the two types of $\mathrm{T}$ cells had a significant impact on prognosis of HCC patients. Amongst, KLRB1 and IL18RAP were final two genes related to both two kinds of T cells and associated with overall survival of the HCC patients.
\end{abstract}

Keywords Hepatocellular carcinoma - Genes · Tumor-infiltrating T cell $\cdot$ Tumor microenvironment $\cdot$ Prognosis

\section{Abbreviations \\ BP Biological process \\ CC Cellular component \\ CTLA-4 Cytotoxic T-lymphocyte-associated protein 4}

$\mathrm{Xin} \mathrm{Lu}$

luxinln@163.com

Extended author information available on the last page of the article 


$\begin{array}{ll}\text { DEGs } & \begin{array}{l}\text { Differentiated expressed genes } \\ \text { ESTIMATE }\end{array} \\ & \begin{array}{l}\text { Estimation of Stromal and Immune cells in Malignant Tumor tissues } \\ \text { using Expression data }\end{array} \\ \text { GO } & \text { Gene Ontology } \\ \text { HCC } & \text { Hepatocellular carcinoma } \\ \text { HR } & \text { Hazard ratio } \\ \text { IFN- } \gamma & \text { Interferon- } \gamma \\ \text { KEGG } & \text { Kyoto Encyclopedia of Genes and Genomes } \\ \text { K-M } & \text { Kaplan-Meier } \\ \text { logFC } & \text { Logarithm of fold changes of genes } \\ \text { MF } & \text { Molecular function } \\ \text { miRNA } & \text { MicroRNA } \\ \text { NK cell } & \text { Natural killer cell } \\ \text { OS } & \text { Overall survival } \\ \text { PD-1 } & \text { Programmed cell death protein 1 } \\ \text { PLC } & \text { Primary liver cancer } \\ \text { PPI } & \text { Protein-protein interaction } \\ \text { TCGA } & \text { The Caner Genome Atlas } \\ \text { TIL } & \text { Tumor-infiltrating lymphocytes } \\ \text { TME } & \text { Tumor microenvironment }\end{array}$

\section{Introduction}

Primary liver cancer (PLC) is the third most common cause of cancer-related mortality $(8.3 \%)$ and the sixth most commonly diagnosed cancer (4.7\%) worldwide in 2020 (Sung et al. 2020). Hepatocellular carcinoma (HCC) is the dominant type, accounting for $75 \%$ to $85 \%$ of all cases of PLC. Surgery is the major treatment for HCC, including local resection and liver transplantation (Galle et al. 2017; Weinmann and Galle 2020). For advanced stage and inoperable patients, however, the recommended treatment plans include systemic therapy with first and second line of multi-kinase inhibitor, such as sorafenib and regorafenib (Bruix et al. 2017; Llovet et al. 2008). Although great progress has been made in diagnostics and therapy during the past decades, HCC remains a terminal disease due to drug resistance and distant metastasis, with a 5-year survival of $18.1 \%$ across all stages and $2.3 \%$ for advanced disease. For patients who suffered from advanced HCC and for whom systemic therapy is the only choice, the 1-year survival rate has been less than $50 \%$ since diagnosis is confirmed (Jemal et al. 2017; Giannini et al. 2015).

In recent years, tumor microenvironment (TME) has been paid much attention by community of medical oncology (Hanahan and Weinberg 2011). Basically, TME consists of extracellular matrix and variety of cells, including fibrocytes, cells derived from vascular structure, and immune cells (Baghban et al. 2020). For liver, it also has various types of cells. They can be classified as two types: one is hepatocellular cell (liver parenchymal cell) and non-parenchymal cells [liver sinusoidal endothelial cells, Kupffer cells, hepatic stellate cells, liver natural killer (NK) cell, DC cell, T/B cell, etc.] (Jiang et al. 2020). These cells 
together build a complex TME in liver and regulate tumor cell biological activities in different ways. The immune compartment in TME of liver is called as tumor-infiltrating lymphocytes (TILs), which is thought to be an innate immune weapon for people to eliminate cancerous cells. $\mathrm{CD}^{+}$and $\mathrm{CD}^{+} \mathrm{T}$ cells are predominant in TILs and early clinical studies found that high level of T-cell infiltration in TME results in a lower recurrence rate and a better survival for patients with HCC (Unitt et al. 2006; Cariani et al. 2012). 18 years ago, Japanese scientists invented a novel algorithm to calculate the ratio of immune cells and stromal cells in malignant tumors via gene expression signature derived from The Cancer Genome Atlas (TCGA). It is called Estimation of Stromal and Immune cells in Malignant Tumor tissues using Expression data (ESTIMATE) algorithm. This algorithm can evaluate and predict the purity of certain kind of tumor (Yoshihara et al. 2013). Numerous clinicians and bioinformaticians have extracted data and initiated research of varieties of solid tumors. Xiang et al. found that increased stromal, immune, and Estimate scores were significantly correlated with better prognosis of HCC patients (Xiang et al. 2021), which is also found in research of other clinical teams (Yu et al. 2021; He et al. 2020a). Nevertheless, existence of T cells cannot avoid tumorigenesis because they can express some immune inhibitory molecules. Activated T cells can express immunosuppressive receptors, such as programmed cell death protein 1 (PD-1) and cytotoxic T lymphocyte-associated protein 4 (CTLA-4). When stimulated effector $\mathrm{T}$ cells attack tumor cells, the immune response is strong at the early time and then turns weaker due to the interactions between PD-1 and its ligands PD-L1 expressed on tumor cells. Then the tumor cells get tolerated to the initial immune response and apoptosis, exhaustion, and phenotype conversion of T cell occurs (Kudo 2019; Alsaab et al. 2017). Different from PD-1, CTLA-4 expressed on matured T cells combines with its ligand CD80/86 on various types of antigen-presenting cells, which can also suppress the immune response initiated by interactions between CD28 of T cells and CD80/86.

The liver is a 'tolerogenic' organ for it is an ideal organ for transplantation surgery and liver allograft is more tolerogenic than that from other organs (Jiang et al. 2020; Zimmermann et al. 1984). This trait of liver tissue is due to the immunosuppressive molecules and cells in liver TME. This immunosuppressive characteristic in liver tissue, however, can also cause immune invasion, which is an essential trigger for initiation and development of HCC (Shlomai et al. 2014). The level of immunosuppression in liver TME is closely related to prognosis of HCC patients. Hence, to reconstruct a strong anti-tumor immunity, the immune landscape of HCC and detailed information of some important immune cells are necessary to be investigated ( $\mathrm{Fu}$ et al. 2019). In this study, we investigated the gene expression pattern associated with tumor-infiltrating non-regulatory $\mathrm{CD}^{+}$ and $\mathrm{CD}^{+} \mathrm{T}$ cells of HCC. The results are expected to provide therapeutic targets for future immunotherapy of HCC. 


\section{Materials and Methods}

\section{Data Acquisition}

The expression data, RNA-sequencing fragments per kilobase of transcript per million mapped reads, and corresponding clinical information from The Cancer Genome Atlas Hepatocellular Carcinoma (TCGA-LIHC) data collection were collected from TCGA GDC database (https://portal.gdc.cancer.gov/). Data acquisition was on 06-03-2021.

\section{Differentially Expressed Genes (DEGs) Identification}

We first transferred and made annotations by Perl (Version 5.30.0) for the probes from expression data according to the annotation files downloaded from Ensemble genome browser 103 (http://ftp.ensembl.org/pub/current_gtf/homo_sapiens/). R studio based on R (v4.0.4) was used to arrange the expression data. R package "limma" (Version 3.46.0) was used. The items in which multiple probes matched to the same gene were filtered and the gene expression was calculated as mean value of all these probes. Stromal scores and immune scores of 374 tumor tissue were calculated using the ESTIMATE package (Version 1.0.13). According to the median level of the stromal/immune score, the DEGs between high stromal/immune scores and low stromal/immune scores were screened using the "limma" packaged with screening criteria of $\mid \log$ fold change $(\mathrm{FC}) \mid>1$ and false discovery rate $(\mathrm{FDR})<0.05$. Then we plotted a heatmap including top 50 up-regulated DEGs and top 50 down-regulated DEGs according to stromal score and immune score, respectively. The package "pheatmap" (Version 1.0.12) was used. Using R package "VennDiagram" (Version 1.6.20), we used two Venn diagrams to illustrate overlapping DEGs of up-regulated and down-regulated genes according to both immune and stromal scores. The overlapping DEGs of up- and down-regulated genes were used in the following analysis.

\section{Functional Enrichment Analysis}

Using R package “org.Hs.eg.db" (Version 3.12.0), the ensemble ID of DEGs of both up- and down-regulated genes were retrieved. The "biological process" (BP), "cellular components" (CC), and "molecular functions" (MF) terms of the Gene Ontology (GO) annotation and the Kyoto Encyclopedia of Genes and Genomes (KEGG) pathways were analyzed via R package of "org.Hs.eg.db," "clusterProfiler" (Version 3.18.1), "enrichplot" (Version 1.10.2), and "ggplot2" (Version 3.3.3) to investigate the functions of the up-regulated and down-regulated overlapping DEGs. The terms with $P<0.05$ were considered to be significant results.

\section{Screening of CD4 ${ }^{+}$and $\mathrm{CD}^{+}{ }^{+}$Cell-Related Genes}

Matrix of reference gene expression in 22 kinds of immune cells was downloaded from CIBERSORTx (https://cibersortx.stanford.edu/) as the reference file (Newman et al. 
2015, 2019). Based on the Cibersort algorithm and "limma" package, the relative abundance $(\%)$ of 22 tumor-infiltrating immune cells in selected HCC samples were estimated (Newman et al. 2015). After that, the correlation coefficient ( $r$ ) between overlapping DEGs and infiltrating abundance of immune cells $\left(\mathrm{CD}^{+}\right.$and $\mathrm{CD}^{+} \mathrm{T}$ cells) was calculated using the Spearman correlation test. Selected $\mathrm{CD} 4^{+}$and $\mathrm{CD} 8^{+} \mathrm{T}$ cell-related genes were identified with correlation coefficient $|r|>0.23$.

\section{Construction of the Protein-Protein Interaction (PPI) Network and Core Genes Selection}

The interactions between $\mathrm{CD}^{+}$and $\mathrm{CD}^{+} \mathrm{T}$ cell-related genes were retrieved from the PPI website STRING database (https://www.string-db.org/) with a PPI score setting as 0.35 and 0.90 , respectively. The PPI network visualization was performed by Cytoscape software (version: 3.6.0). Then Perl language and R were used to summary the top 30 core genes in retrieved PPI network of $\mathrm{CD}^{+}$and $\mathrm{CD}^{+} \mathrm{T}$ cell, respectively.

\section{Construction of the MicroRNA (miRNA)-Target Gene Networks}

To predict miRNA-target gene interactions, core genes in PPI networks of $\mathrm{CD}^{+}$and $\mathrm{CD}^{+} \mathrm{T}$ cell were input to miRwalk 3.0 (http://mirwalk.umm.uni-heidelberg.de/), a miRNA-target gene prediction database. For settings of parameters, the miRNA-target gene interactions with a score $>0.95$ and those that existed in both the TargetScan and miRDB databases were selected. The miRNA-target gene network for both $\mathrm{CD}^{+}$and $\mathrm{CD}^{+}$cells were visualized in Cytoscape.

\section{Survival Analysis}

The clinical data and expression data of $\mathrm{CD}^{+}$and $\mathrm{CD}^{+} \mathrm{T}$ cell-related genes were integrated using Perl language and "limma" package. The prognosis-related genes were summarized by function " $\operatorname{coxph}()$ " and demonstrated with forest plots. The overall survival (OS) and OS status in the clinical data were used to perform survival analysis via R package "survival" (Version 3.2-7) and "survminer" (Version 0.4.8). In this process, the immune cell-related genes were divided into low-expression group and highexpression group based on the median level of gene expression, together with a logrank statistical test. The cut-off was set as a $P$ value $<0.05$ to select the significantly prognosis-related genes in $\mathrm{CD}^{+}$and $\mathrm{CD}^{+}$group and then Kaplan-Meier $(\mathrm{K}-\mathrm{M})$ survival curves were plotted.

\section{Results}

\section{Differentiated Expression Gene was Collected Based on Stromal Scores}

The total number of samples in TCGA-LIHC data is 424, 374 of which were samples of HCC, while the other 50 samples were normal liver tissue. Via 
ESTIMATE algorithm, stromal scores and immune scores were successfully calculated in all $374 \mathrm{HCC}$ samples. The higher the stromal/immune scores were, the higher proportions of stromal/immune cells in the samples were. According to the stromal scores in all HCC samples, the median score was - 659.4628. The number of samples whose scores higher than the median level was 187, the same as that of samples with scores no more than the median level. The gene expression data of $374 \mathrm{HCC}$ samples have been further processed by removing replicated genes and genes with an average expression no more than 0.1 . Through calculation of difference of gene expression, final 1891 genes were defined as DEGs. Among, 1670 genes were up-regulated in samples with a higher stromal score, whereas 221 genes were down-regulated in samples with a higher stromal score. According to the value of logarithm of FCs (logFC) of genes, top 50 up-regulated and top 50 down-regulated genes are plotted in the heatmap (Supplementary Fig. 1) and summarized in Supplementary Table 1.

\section{Differentially Expressed Genes were Collected Based on Immune Scores}

According to the calculation of immune scores in 374 HCC samples, the median value was 432.3971. The number of samples with scores no more than median level equaled to that of samples with scores exceeding the median value (187 for each group). Based on the selection criteria aforementioned, final 1579 genes were designated as DEGs. Among, 1418 genes were up-regulated in samples with immune scores higher than median level, while 161 genes were down-regulated in samples whose immune scores were less than median value. The top 50 upregulated and top 50 down-regulated genes are illustrated in heatmap (Supplementary Fig. 2) and the information of these genes are summarized in Supplementary Table 2 .

\section{Overlapping Up-regulated and Down-regulated Genes According to Both Stromal and Immune Scores were Analyzed in GO and KEGG Database}

According to the results mentioned above, genes which were up-regulated based on both stromal and immune scores and which were down-regulated are collected and illustrated in Venn diagrams (Supplementary Fig. 3a, b). The number of overlapping up-regulated DEGs was 1076 and the number of overlapping down-regulated DEGs was 62. Table 1 summarizes the top 50 overlapping upregulated-down-regulated DEGs.

The $\log F C$ value in this table was calculated as the mean of the $\log F C$ based on stromal score and immune score. GO and KEGG analysis of these overlapping genes are illustrated in Supplementary Figs. $4 \mathrm{a}$ and $\mathrm{b}$ and $5 \mathrm{a}$ and b, respectively. In $\mathrm{GO}$ analysis, the top 10 highest enriched genes with functions of $\mathrm{BP}, \mathrm{CC}$, and MF were showed. In the picture of KEGG, the top 30 highest enriched gene pathways were showed. Most of them were associated with immune response. 
Table 1 Overlapping up-regulated and down-regulated DEGs based on both stromal and immune scores

\begin{tabular}{|c|c|c|c|}
\hline Up-regulated gene & $\log \mathrm{FC}$ & Down-regulated gene & $\log \mathrm{FC}$ \\
\hline TRARG1 & 7.666054729 & KLK4 & -2.890539921 \\
\hline CRTAC1 & 5.532685203 & RGSL1 & -2.205440106 \\
\hline IGFN1 & 5.265016334 & RHBDL3 & -2.184229516 \\
\hline C16orf89 & 4.865872612 & LINC02587 & -2.144128848 \\
\hline IGKV1D-42 & 4.825363156 & AP000593.3 & -2.023942443 \\
\hline IGLV5-45 & 4.512147135 & AURKBP1 & -1.894678211 \\
\hline IGHV1-58 & 4.441232443 & AC007277.1 & -1.891447694 \\
\hline IGLV10-54 & 4.440730135 & AL590483.2 & -1.881354742 \\
\hline IGKV2D-40 & 4.432250477 & C1QTNF3 & -1.860001267 \\
\hline IGKV6-21 & 4.431686791 & RASL10B & -1.791626005 \\
\hline IL11 & 4.009377903 & RHBG & -1.780671072 \\
\hline OMG & 3.9775617 & PAGE4 & -1.778360546 \\
\hline IGHV3-73 & 3.931511939 & AC104088.1 & -1.750003438 \\
\hline CCL19 & 3.858908479 & ACTN2 & -1.726351182 \\
\hline IGLV3-16 & 3.791071906 & CTNNA2 & -1.717561344 \\
\hline CHIT1 & 3.764407355 & KCNU1 & -1.691739858 \\
\hline CHRNA1 & 3.747690595 & AC026765.3 & -1.685375046 \\
\hline IGHV1-2 & 3.724027271 & AC011747.1 & -1.585204355 \\
\hline $\mathrm{CR} 2$ & 3.71985571 & NOTUM & -1.557252788 \\
\hline IGLC7 & 3.717952082 & LGR5 & -1.556438279 \\
\hline IGLV2-8 & 3.714584762 & AC069294.1 & -1.541662443 \\
\hline IGKV3D-15 & 3.64296302 & AL163953.1 & -1.505394178 \\
\hline IGLV4-69 & 3.61053471 & ABHD1 & -1.485544254 \\
\hline IGHV3-11 & 3.574892854 & LINC01970 & -1.484943844 \\
\hline CRYBB1 & 3.543085965 & GLUL & -1.445004555 \\
\hline TNNT3 & 3.507231587 & GLULP4 & -1.409807598 \\
\hline IGHV3-64 & 3.45118729 & MIR325HG & -1.405286019 \\
\hline IGHV3-71 & 3.444000853 & ACSL6 & -1.401318749 \\
\hline IGLV8-61 & 3.440634419 & DNAJB3 & -1.394672128 \\
\hline DES & 3.423964824 & SP5 & -1.359215973 \\
\hline AC135068.1 & 3.417111295 & LINC01124 & -1.341499193 \\
\hline IGHV3-15 & 3.40543775 & AC010501.2 & -1.332176671 \\
\hline IGKV1-9 & 3.361738473 & AC114947.1 & -1.331232992 \\
\hline IGHV3-33 & 3.349048911 & AC113404.1 & -1.328616526 \\
\hline IGHV1-69 & 3.328530619 & GREB1 & -1.31633006 \\
\hline IGHV2-70 & 3.312158823 & MTND4LP30 & -1.316294715 \\
\hline IGKV1D-13 & 3.296648953 & AC008549.1 & -1.303165529 \\
\hline WNT2 & 3.288090828 & AC007406.2 & -1.2826021 \\
\hline IGHV3-19 & 3.277470772 & AC013244.1 & -1.268139454 \\
\hline IGHV2-70D & 3.276646515 & SRARP & -1.259680291 \\
\hline IGHV1-24 & 3.275489277 & C5orf66 & -1.258706959 \\
\hline IGHV2-26 & 3.264057216 & ASB4 & -1.251581711 \\
\hline
\end{tabular}


Table 1 (continued)

\begin{tabular}{llll}
\hline Up-regulated gene & $\operatorname{logFC}$ & Down-regulated gene & $\operatorname{logFC}$ \\
\hline IGKV3D-11 & 3.239636373 & TBX3 & -1.243487135 \\
PLA2G2D & 3.225196379 & RNU6-46P & -1.237461012 \\
MS4A1 & 3.223865765 & AC005841.1 & -1.227513059 \\
IGHG2 & 3.214812763 & FAM169A & -1.217327844 \\
NDNF & 3.203978929 & TECTB & -1.21055518 \\
IGLV2-11 & 3.199379915 & H4C10P & -1.198960549 \\
IGKV2-30 & 3.191285367 & ACTG1P25 & -1.197484908 \\
IGLV1-40 & 3.189484969 & AC010531.5 & -1.19431992 \\
\hline
\end{tabular}

$D E G s$ differentially expressed genes, $F C$ fold change

\section{Genes Associated with Tumor-Infiltrating $\mathrm{CD4}^{+}$and $\mathrm{CD8}^{+} \mathrm{T}$ Cell were Identified by CIBERSORTx Algorithm}

Using Spearman's correlation test, the correlation coefficients $(r)$ of overlapping DEGs with the abundance ratio of $\mathrm{CD}^{+} \mathrm{T}$ cell and $\mathrm{CD}^{+} \mathrm{T}$ cell were successfully calculated. Genes with $|r|>0.23$ were included. By this criteria, $103 \mathrm{CD}^{+} \mathrm{T}$ cellrelated genes and $407 \mathrm{CD}^{+} \mathrm{T}$ cell-related genes were collected. Eight genes were found to be correlated with both kinds of $\mathrm{T}$ cells and this was illustrated via Venn diagram in Fig. 1. Based on the abundance ratio of several main immune cells via Cibersort algorithm, barplots of the proportion of these immune cells are demonstrated in Supplementary Fig. 6. In this picture, it is found that $\mathrm{CD}^{+}$and $\mathrm{CD}^{+}$ $\mathrm{T}$ cells taking up a large proportion in tumor-infiltrating immune cells in TME of

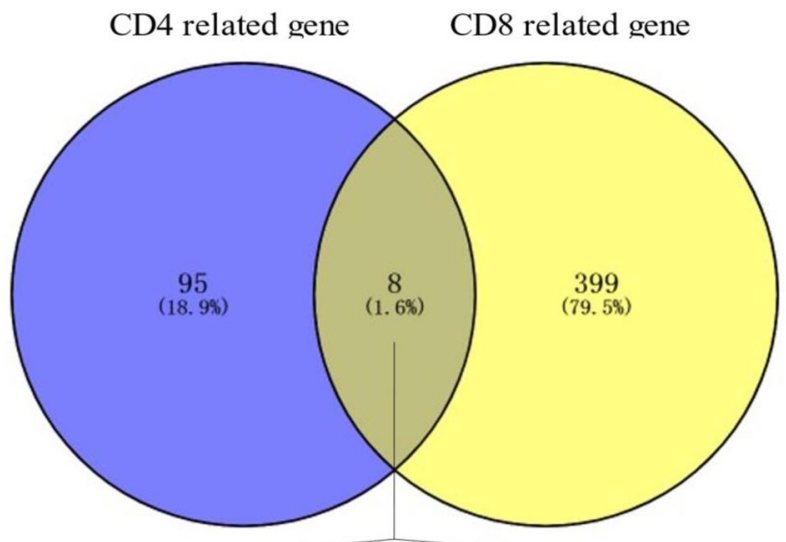

KLRB1, IL18RAP, CD69, CD226, PTGDR, TAF5LP1, LINC01679, TMC8

Fig. 1 Number of $\mathrm{CD}^{+}$and $\mathrm{CD}^{+} \mathrm{T}$ cell-related genes and the gene symbols of eight overlapping genes in HCC samples. HCC hepatocellular carcinoma 
HCC. In this plot, only samples with $P$-value $<0.05$ will be included to be used for analysis.

\section{Core Genes Related to Tumor-Infiltrating CD4 ${ }^{+}$and $\mathrm{CD8}^{+} \mathrm{T}$ Cell in PPI Network were Retrieved}

Based on results of $\mathrm{CD}^{+}$and $\mathrm{CD}^{+} \mathrm{T}$ cell-related genes, PPI networks of these genes were plotted, which are shown in Fig. 2a and b. Considering the number of genes and the effect of visualization of the network, the interaction confidence of $\mathrm{CD}^{+} \mathrm{T}$ cell-related genes was set as 0.35 , while that of $\mathrm{CD} 8^{+} \mathrm{T}$ cell-related genes was set as 0.90 . Of notice, all the genes visualized in these pictures were all upregulated DEGs according to the DEG analysis. Based on PPI data, the number of interactions for each node were summarized and core genes in PPI network were defined as the top $30 \mathrm{CD}^{+}$and $\mathrm{CD}^{+} \mathrm{T}$ cell-related genes according to the number of interactions for each node and the plot is illustrated in Fig. 3a, b.

\section{miRNA-Target Gene Interaction Network of Core Genes Related to Tumor-Infiltrating CD4 ${ }^{+}$and $\mathrm{CD8}^{+} \mathrm{T}$ Cell were Visualized}

The prediction of miRNA-target gene interaction was conducted by software miRwalk 3.0. Networks of predicted miRNA-gene interactions with $\mathrm{CD}^{+}{ }^{+} \mathrm{T}$ cells and $\mathrm{CD}^{+} \mathrm{T}$ cells are illustrated in Supplementary Fig. 7a and b, respectively.

\section{Prognosis-Related Genes Associated with Tumor-Infiltrating $\mathrm{CD}^{+}$and $\mathrm{CD}^{+} \mathrm{T}$ Cell were Found in HCC Samples}

Candidate genes for survival analysis came from $\mathrm{CD}^{+}$and $\mathrm{CD} 8^{+} \mathrm{T}$ cell-related genes. Cox regression method was used for OS analysis. In total, six genes of CD4 ${ }^{+}$ $\mathrm{T}$ cells were found significantly related to OS of HCC patients and the forest plot has told that all these genes were beneficial to HCC patients since that the expressions of these genes increased in patients with a better prognosis [hazard ratio $(\mathrm{HR})<1$ ]. Moreover, 30 genes of $\mathrm{CD}^{+} \mathrm{T}$ cell-related genes were found significantly related to $\mathrm{OS}$ of $\mathrm{HCC}$ patients. Corresponding forest plot told that 21 genes were high-risk genes whose expression increased in patients with a shorter OS (HR $>1)$ and the other nine genes were beneficial to HCC patients (Supplementary Fig. 8a, b). Via $\mathrm{K}-\mathrm{M}$ analysis, final four genes related to $\mathrm{CD}^{+} \mathrm{T}$ cells and final four genes relate to $\mathrm{CD} 8^{+} \mathrm{T}$ cells were found significantly associated with prognosis (OS) of HCC patients. Among them, KLRB1 and IL18RAP were two overlapping genes not only influential to HCC patients' OS but also related to both $\mathrm{CD}^{+}{ }^{+}$and $\mathrm{CD} 8^{+} \mathrm{T}$ cell. And both of these genes were clinically beneficial to HCC patients. Their high expressions tended to result in a relatively long OS for HCC population. Survival curves of these two genes are shown in Fig. $4 \mathrm{a}$ and $\mathrm{b}$ and the other prognosis-related genes are illustrated in Supplementary Fig. 9a and b. 


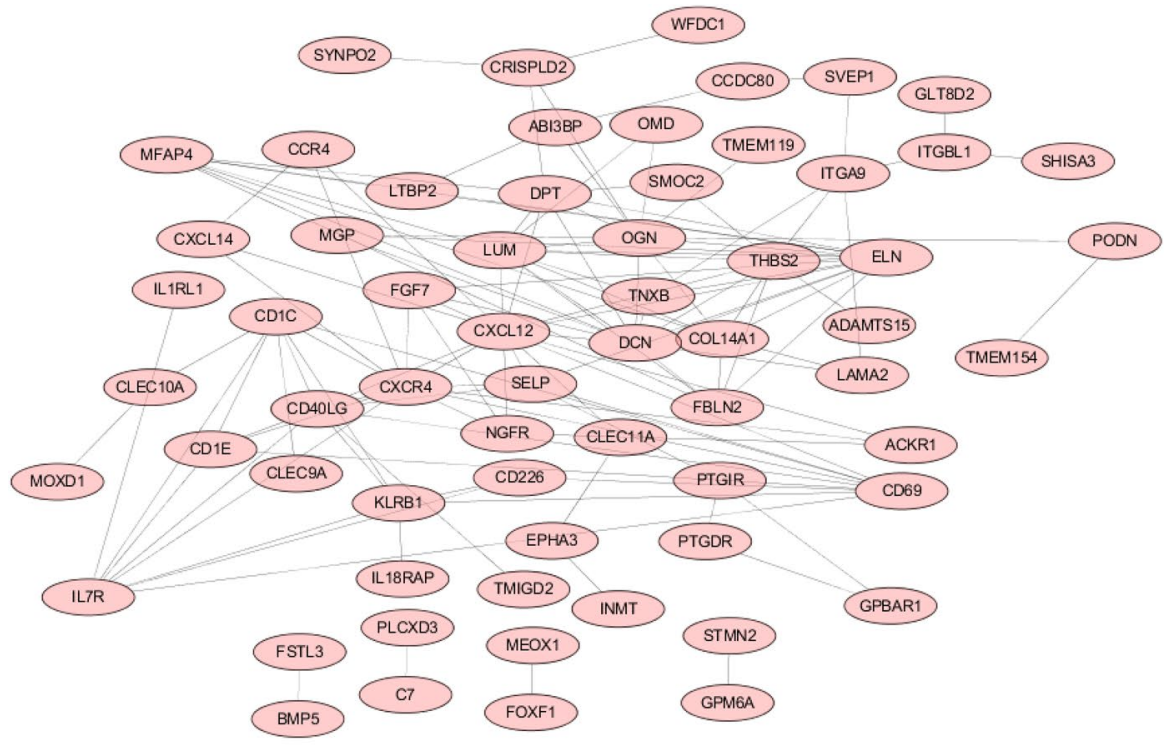

(a)

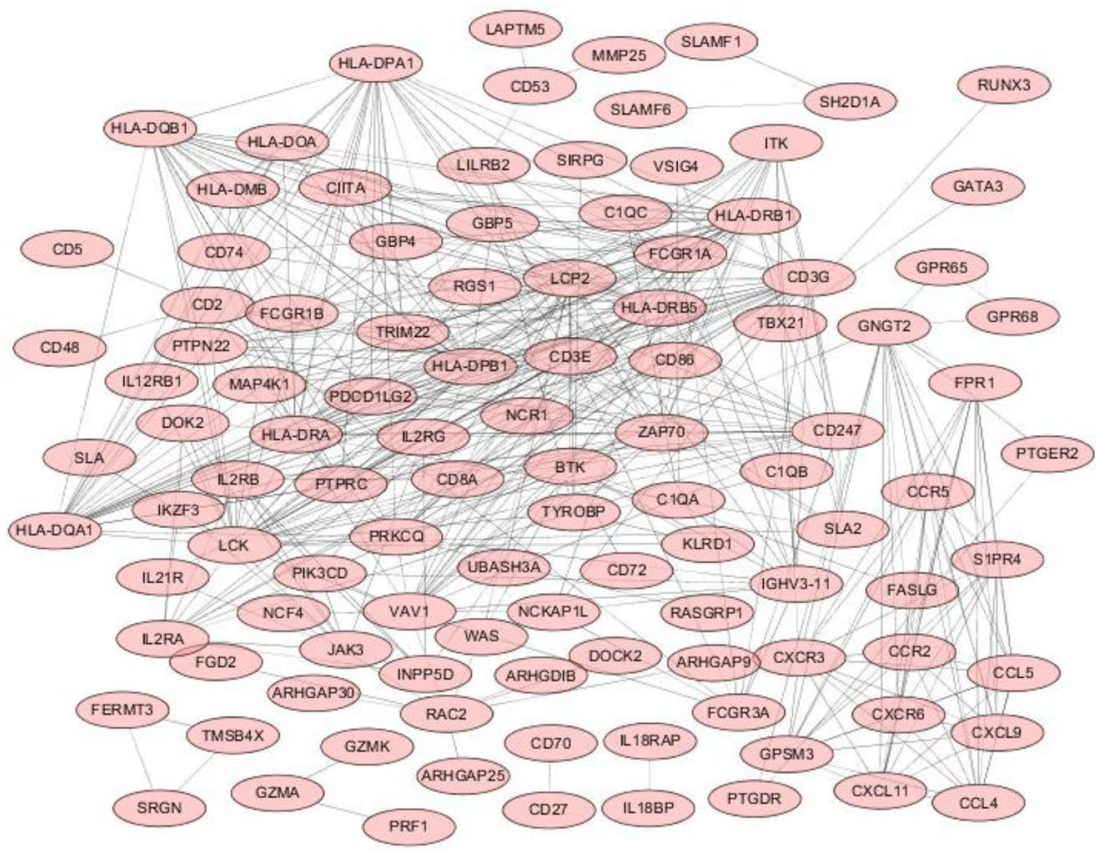

(b)

Fig. 2 Protein-protein network of $\mathrm{CD} 4^{+} \mathrm{T}$ cell-related genes (a) and $\mathrm{CD} 8^{+} \mathrm{T}$ cell-related genes $(\mathbf{b})$ 


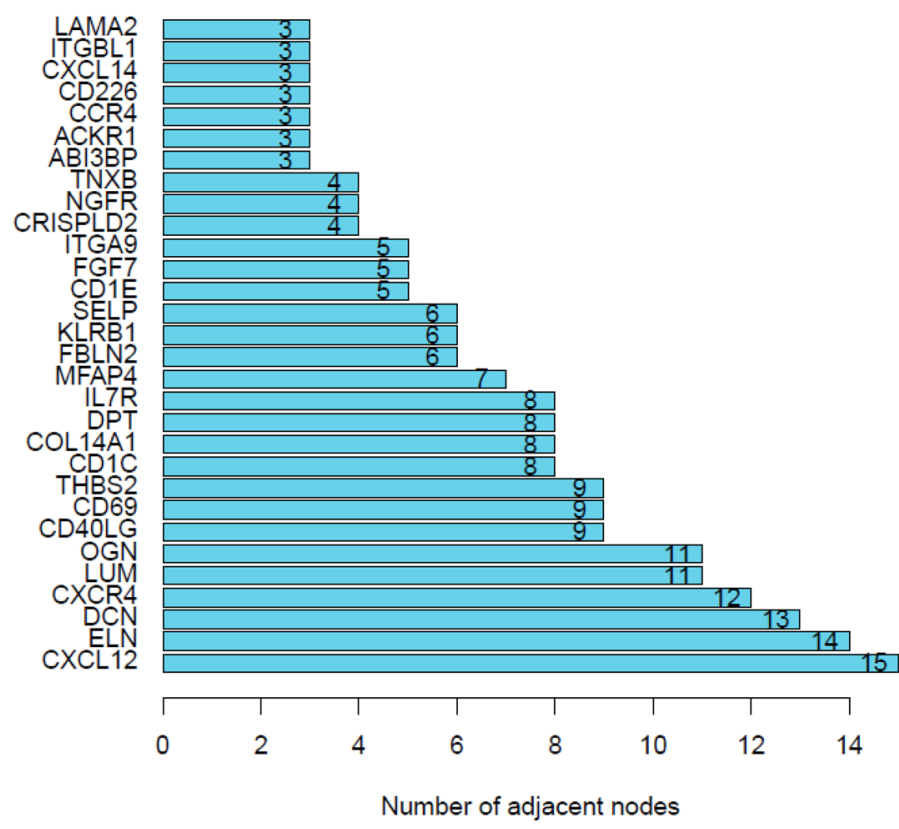

(a)

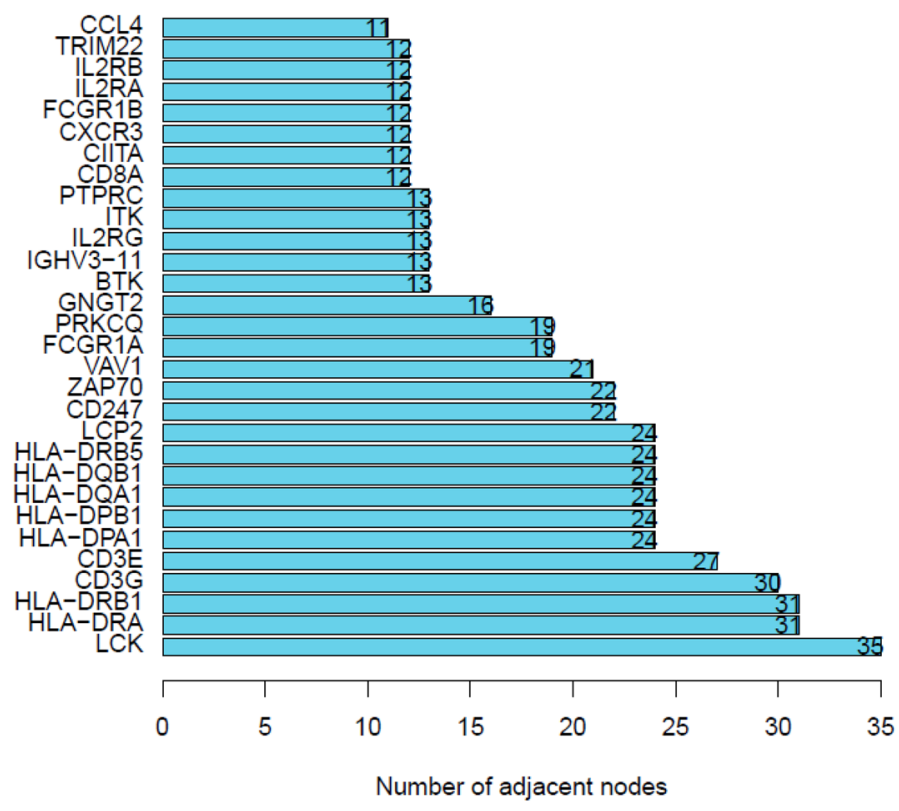

(b)

Fig. 3 Core genes in PPI networks of T cell-related genes. a Collected top 30 core genes related to CD4 ${ }^{+}$ $\mathrm{T}$ cells and $\mathbf{b}$ demonstrated top 30 genes associated with $\mathrm{CD} 8^{+} \mathrm{T}$ cells. PPI protein-protein interactions 


\section{Discussion}

In our study, 1579 DEGs in HCC samples between high stromal/immune scores were identified, $89.8 \%$ of which were up-regulated genes and $10.2 \%$ of which were down-regulated genes. $75.9 \%$ of the whole DEGs were overlapping up-regulated genes based on both stromal and immune scores, while $38.5 \%$ were overlapping down-regulated genes. Among these overlapping DEGs, immune-related items were the major elements in top 10 enriched biological function, CC, and MFs. This suggested that DEGs played an important role in immune regulation. And this stimulated us to further explore the relationship of genes with $\mathrm{CD}^{+}$and $\mathrm{CD}^{+} \mathrm{T}$ cells. Of the 1138 overlapping DEGs, $9.1 \%$ of them were correlated with relative abundance of $\mathrm{CD}^{+} \mathrm{T}$ cells and $35.8 \%$ were associated with that of $\mathrm{CD} 8^{+}$ $\mathrm{T}$ cells. Among these genes, eight of them were related with both of these two kinds of $\mathrm{T}$ cells and the proportion was around 1.6\%. Moreover, via summarizing the immune cell proportion in different HCC samples, we found that CD4 ${ }^{+}$ and $\mathrm{CD}^{+} \mathrm{T}$ cells were major subtypes of immune cell family, which reassured the reasonability of investigating immune-related genes. Speaking of the PPI networks, 30 core genes related to $\mathrm{CD}^{+} \mathrm{T}$ cells and $30 \mathrm{CD}^{+} \mathrm{T}$ cell-related genes were designated. For miRNA-target networks, genes targeted by existing miRNAs took up more than $50 \%$ in two groups, which indirectly proved their core status in PPI network. In prognosis analysis, both of the Cox regression and K-M survival analysis found that only four genes related to $\mathrm{CD} 4^{+} \mathrm{T}$ cells and four genes correlated with $\mathrm{CD}^{+} \mathrm{T}$ cells were significantly prognosis-related factors for HCC patients. KLRB1 and IL18RAP were two overlapping genes not only related to both $\mathrm{CD}^{+}{ }^{+}$and $\mathrm{CD}^{+}{ }^{+} \mathrm{T}$ cells but also had a protective effect for $\mathrm{HCC}$ patients. Their high expression tended to appear in patients with a longer OS.

The immune tolerance and immune evasion in the liver usually causes initiation of HCC or liver metastasis from other types of solid tumors (Shlomai et al. 2014). Chronic infection of hepatitis $B$ virus and hepatitis $C$ virus and toxic injuries derived from alcohol and aflatoxin are typical stimulators of HCC. These risk factors are likely to induce immunosuppression through host-viral interaction or chronic inflammation (Woller et al. 2015). Briefly, elements of immunosuppression include inhibitory cytokines or chemokines, defects of antigen presentation, and immunosuppression cells in liver microenvironment (Hato et al. 2014; Zhang et al. 2010). In addition to HCC, immune tolerance can also contribute to liver metastasis of other solid malignancies. Liver metastasis tend to weaken the effect of systemic therapy, such as immune checkpoint inhibitors, including PD-1 and CTLA-4 antibodies. It is in part associated with a decreased infiltration of CD8 ${ }^{+}$ $\mathrm{T}$ cell (Tumeh et al. 2017). As a result, more information of immune landscape of HCC microenvironment should be extracted and the methods to reverse the repressive and tolerant immunity of liver microenvironment are badly needed to be found.

In our study, we found that KLRB1 and IL18RAP were up-regulated in the HCC samples with a higher than median immune/stromal score and the Spearman correlation coefficient $(r)$ of the two genes were all more than 0.23. Patients with 

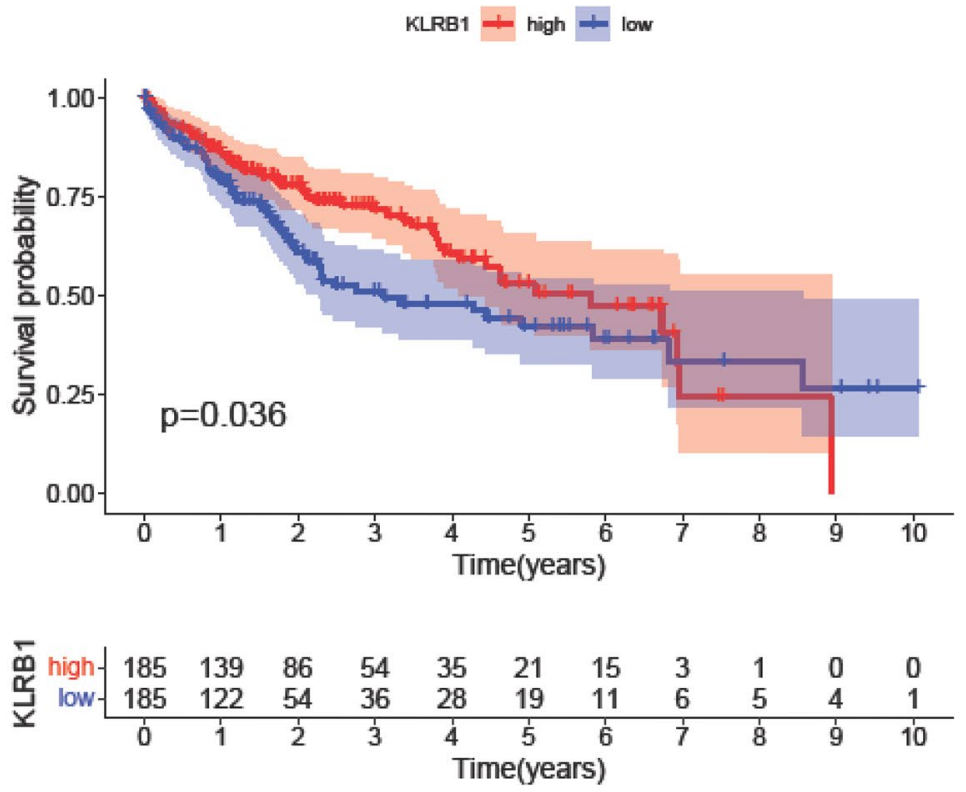

(a)

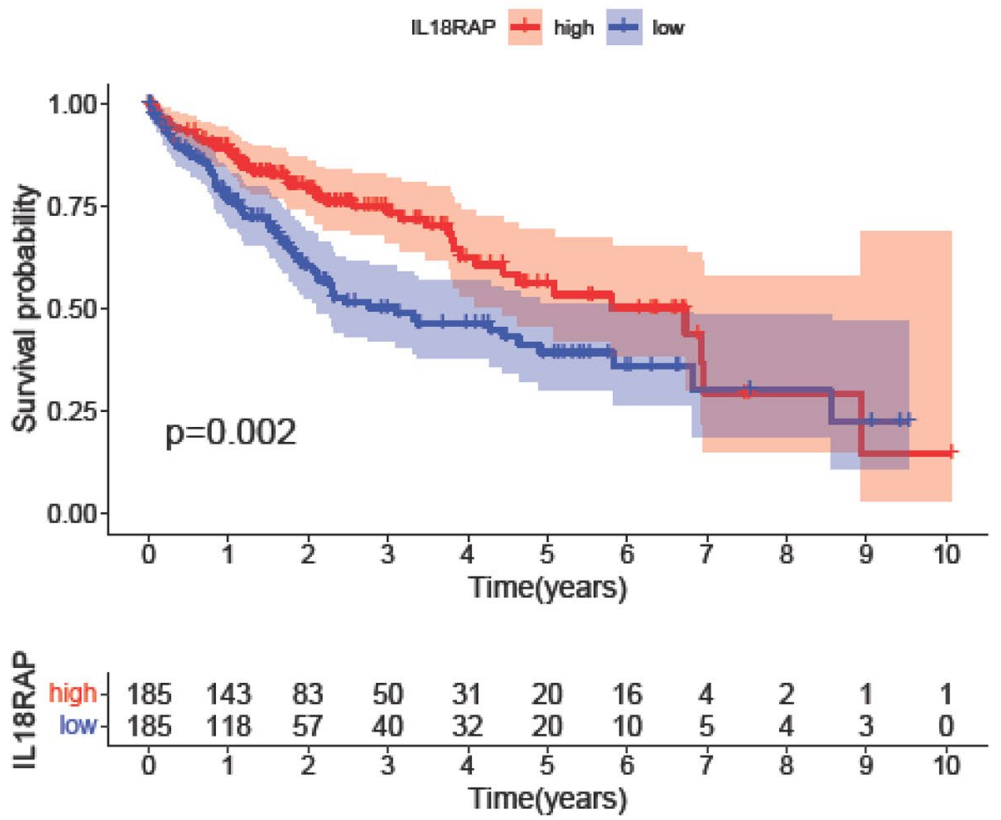

(b)

Fig. 4 Two genes, KLRB1 (a) and IL18RAP (b) were two genes not only related to abundance of both $\mathrm{CD}^{+}$and $\mathrm{CD} 8^{+} \mathrm{T}$ cells but also significantly associated with prognosis of HCC patients. Both of them were beneficial to $\mathrm{HCC}$ patients with a $\mathrm{HR}<1$. $H C C$ hepatocellular carcinoma, $H R$ hazard ratio 
a longer OS had a relatively high expression of KLRB1 and IL18RAP. Speaking of survival, higher expression of these two genes corresponds to a longer survival of patients with HCC. Several researches had found the relationship between solid tumors and these two genes.

KLRB1, whose full name is killer cell lectin-like receptor subfamily B 1 and is also named as CD161, is a member of NKRP1 subfamily which codes NKRP1A molecule (Mesci et al. 2006). This molecule is a kind of type II transmembrane glycoprotein whose molecular weight ranges between 40 and $44 \mathrm{kDa}$ (Lanier et al. 1994; Poggi et al. 1996). NKRP1A has an extracellular region, including a C-type lectin domain, a transmembrane region, and an intracellular region, including an immunoreceptor tyrosine-based inhibitory motif (Lanier et al. 1994; Rosen et al. 2008). NKRP1A is mainly expressed in NK cells as well as T cells secreting IL-17, CD8 ${ }^{+}$T cells, and $\gamma \delta \mathrm{T}$ cells (Poggi et al. 1998; Maggi et al. 2010; Turtle et al. 2011). Functions of NKRP1A are different in different immune cells. Scientists have found that NKRP1A can inhibit cytotoxicity and interferon- $\gamma$ (IFN- $\gamma$ ) secretion of NK cells (Aldemir et al. 2005). Different from this, NKRP1A on T cells on one hand enhances expression of IL-17, tumor necrosis factor- $\alpha$ (TNF)- $\alpha$, and IFN- $\gamma$ and on the other hand reduces the production of TNF in $\mathrm{CD}^{+} \mathrm{T}$ cells (Rosen et al. 2008; Germain et al. 2011). Clinical scientists have also focused on this gene in studies of solid malignancies. A clinical study led by Zhang et al. found that KLRB1 was a potential prognostic marker for esophageal squamous cell carcinoma (Zhang et al. 2020). In a study led by research team from France, receptor-ligand pair of CD161-lectin-like transcript 1 (LLT) has a positive impact on survival of patients suffered from non-small cell lung carcinoma $(P=0.016, \mathrm{HR}=0.88)$ (Braud et al. 2018).

IL18RAP, short for interleukin 18 receptor accessory protein, expresses the accessory $\beta$-subunit of the heteromeric receptor for IL18, a cytokine regulating the immunity by its pro-inflammatory effect and promoting tumor initiation and progression (Kaplanski 2018). In depth, IL18RAP strengthens the combining force between IL18 and its receptor, which has a positive effect on IL18 signaling (Kaplanski 2018; Hedl et al. 2014). This so-called IL18-IL18RAP axis has been found to make a big difference in cancers (Lin et al. 2020). One study suggested that IL18RAP was helpful in tumorigenesis of NKT cell lymphoma. In this disease, the protein encoded by this gene could activate NKT cell, while knockdown of this gene focus could reduce the proliferation of NKT cancer cell by arrest at G0/G1 and G2/M phases. This is a strong evidence of association between IL18RAP and NKT cell lymphoma $\left(P<1.0 \times 10^{-6}\right)$. Another study found that individuals carrying IL18RAP single-nucleotide polymorphisms rs917997 were more vulnerable to gastric cancer or precancerous disease (odds ratio $=1.83$ ) (Wang et al. 2016). The study also mentioned that this gene polymorphism was significantly associated with ulcerative colitis and Crohn's disease, which indicated the pro-inflammatory role of IL18RAP in these inflammatory gastrointestinal morbidities (Wang et al. 2016; Zhernakova et al. 2008). However, whether this gene affected other types of solid malignancies were not clear. Since HCC usually developed on the basis of chronic liver inflammation, IL18RAP has a high likelihood to play a key role in microenvironment of HCC. 
Although these two genes have a significant clinical meaning, merely KLRB 1 was illustrated in top 30 core genes associated with $\mathrm{CD}^{+}{ }^{+} \mathrm{T}$ cells according to the PPI networks. IL18RAP was not illustrated either in PPI network of core genes associated with $\mathrm{CD}^{+}{ }^{+} \mathrm{T}$ cells or $\mathrm{CD}^{+} \mathrm{T}$ cells. It is likely that there is no direct link between gene interaction and prognosis impact of genes. The former one is the issue related with molecular signaling pathways, whereas the latter one emphasizes on the relationship between genes' expression and patients' survival. The same was occurred in network of miRNA-target gene network. Still, we would like to further explore the potential signal pathways of these genes, especially related regulatory molecules, such as miRNA.

The other four genes related either to $\mathrm{CD}^{+}{ }^{+}$or $\mathrm{CD} 8 \mathrm{~T}$ cells are also pivotal in varieties of biochemical reactions. GPR182, short for G Protein-Coupled Receptor 182 , is a member of $\mathrm{G}$ protein-coupled receptors (Kapas et al. 1995). It is highly expressed in tumor endothelial cells of zebrafish and mouse (Xiao et al. 2014). Especially, this gene is enriched in myeloid leukemia of zebrafish, suggesting that GPR182 plays a role in hematopoiesis no matter in healthy people or patients (Alghisi et al. 2013; Kwon et al. 2020). What's more, one study found that GPR182 was produced in varieties of lymphatic endothelial cells that distributed in lymph nodes, skin, and tissue of intestine (Le Mercier et al. 2021). SPOCD1, abbreviation of "spen paralogue and orthologue C-terminal domain containing 1," is a brandnew gene expressing a kind of the transcription factor S-II family (Kimura et al. 2006). At the beginning, this molecule was found to combine with testis protein phosphatase 1, a eukaryotic serine/threonine-specific phosphatase regulating cell signaling (Fardilha et al. 2011). Liu et al. found that SPOCD1 was significantly upregulated in ovarian cancer and was correlated with a higher tumor grade and staging. The possible mechanism is activating PI3K/AKT pathway which slows down the apoptosis of ovarian cancer cells (Liu et al. 2020). Liang et al. suggested that this molecule, as a pro-oncogenic factor, was highly expressed in osteosarcoma tissue, especially the highly invasive phenotype (Liang et al. 2018). Some other studies also demonstrated that SPOCD1 was highly expressed bladder cancer and gastric cancer, stimulating cell proliferation, migration, and invasiveness (van der Heijden et al. 2016; Zhu et al. 2017). As for IL7R, interleukin-7 receptor, it plays an important role in tumor immunity through the conventional JAK/STAT pathway as well as endogenous competitive regulating RNA networks (Fan et al. 2018). A study illustrated that it is significantly related to immune cells, including CD8 T cell and Treg and somatic mutations (He et al. 2020a, b). This conclusion was also supported by Yin's research, which said that high expression of IL7R can enhance anti-tumor immunity and indicate a better prognosis of HCC patients (Yin et al. 2020).

Despite some novel findings in our study, some limitations have to be noticed: (1) for Cibersort algorithm, HCC samples eligible for analysis of abundance of immune cells were no more than 100 and a larger sample size of eligible samples for Cibersort algorithm for data integration of immune cells is necessary; (2) due to heterogeneous molecular phenotypes of regulatory $\mathrm{T}$ cells, we cannot put this subgroup of $\mathrm{T}$ cells in either $\mathrm{CD}^{+}{ }^{+}$or $\mathrm{CD}^{+} \mathrm{T}$ cell to complete further analysis and the relationship of relative abundance of regulatory $T$ cells and genes cannot be clarified in our study; (3) since survival analysis is a clinical issue, the association between the mentioned genes and 
the prognosis of HCC patients is necessary to be proved in future clinical trials; and (4) down-regulated genes in PPI and miRNA-target gene network will be encouraged to explore in future investigation.

\section{Conclusion}

To conclude, DEGs between high and low stromal/immune scores were determined. Genes related to tumor infiltration $\mathrm{T}$ cells were also be identified. Among, a total of six genes related to $\mathrm{CD}^{+}$and/ or $\mathrm{CD}^{+}{ }^{+} \mathrm{T}$ cells had a significant impact on OS of HCC patients, in which KLRB1 and IL18RAP were two genes related to both of these two types of immune cells in HCC samples.

Supplementary Information The online version contains supplementary material available at https://doi. org/10.1007/s10528-021-10175-3.

Acknowledgements The authors of this manuscript sincerely appreciate the efforts of all researchers who have contributed the data to the public databases of TCGA, miRwalk, ESTIMATE, and Cibersort. The interpretation and reporting of these data are the sole responsibility of the authors.

Author Contributions XL conceived, designed, and supervised the overall study. ZZ, CW, and PC conducted data processing and computational analyses. ZZ drafted the manuscript. All authors read and approved the final manuscript.

Funding This work was supported by International Science and Technology Cooperation Projects (2016YFE0107100 and 2015DFA30650) and CAMS Clinical and Translational Medicine Research Funds (2019XK320006).

Data Availability The Cancer Genome Atlas Hepatocellular Carcinoma (TCGA-LIHC) data collection were downloaded from TCGA GDC website (https://portal.gdc.cancer.gov/); data of stromal and immune score were using ESTIMATE algorithm, which are originated from officiate website of ESTIMATE (https://bioinformatics.mdanderson.org/estimate/); archives of gene symbols were downloaded from Ensemble genome browser 103 (http://ftp.ensembl.org/pub/current_gtf/homo_sapiens/); Cibersort algorithm and its Appendix reference data from official website of CIBERSORTX (https://cibersortx.stanf ord.edu/). The raw materials of PPI network and miRNA-target gene networks were produced via official websites of STRING (https://www.string-db.org/) and miRwalk 3.0 (http://mirwalk.umm.uni-heidelberg. $\mathrm{de} /)$.

\section{Declarations}

Conflict of interest The authors declare that they have no competing interests.

Ethical Approval Not applicable.

Consent for Publication All authors have provided consent for the publication of this manuscript in Biochemical Genetics.

Open Access This article is licensed under a Creative Commons Attribution 4.0 International License, which permits use, sharing, adaptation, distribution and reproduction in any medium or format, as long as you give appropriate credit to the original author(s) and the source, provide a link to the Creative Commons licence, and indicate if changes were made. The images or other third party material in this article are included in the article's Creative Commons licence, unless indicated otherwise in a credit line to the material. If material is not included in the article's Creative Commons licence and your intended use is 
not permitted by statutory regulation or exceeds the permitted use, you will need to obtain permission directly from the copyright holder. To view a copy of this licence, visit http://creativecommons.org/licen ses/by/4.0/.

\section{References}

Aldemir H, Prod'homme V, Dumaurier MJ et al (2005) Cutting edge: lectin-like transcript 1 is a ligand for the CD161 receptor. J Immunol 175(12):7791-7795

Alghisi E, Distel M, Malagola M et al (2013) Targeting oncogene expression to endothelial cells induces proliferation of the myelo-erythroid lineage by repressing the Notch pathway. Leukemia 27(11):2229-2241

Alsaab HO, Sau S, Alzhrani R et al (2017) PD-1 and PD-L1 checkpoint signaling inhibition for cancer immunotherapy: mechanism, combinations, and clinical outcome. Front Pharmacol 8:561

Baghban R, Roshangar L, Jahanban-Esfahlan R et al (2020) Tumor microenvironment complexity and therapeutic implications at a glance. Cell Commun Signal 18(1):59

Braud VM, Biton J, Becht E et al (2018) Expression of LLT1 and its receptor CD161 in lung cancer is associated with better clinical outcome. Oncoimmunology 7(5):e1423184

Bruix J, Qin S, Merle P et al (2017) Regorafenib for patients with hepatocellular carcinoma who progressed on sorafenib treatment (RESORCE): a randomised, double-blind, placebo-controlled, phase 3 trial. Lancet 389(10064):56-66

Cariani E, Pilli M, Zerbini A et al (2012) Immunological and molecular correlates of disease recurrence after liver resection for hepatocellular carcinoma. PLoS ONE 7(3):e32493

Fan Y, Nan Y, Huang J et al (2018) Up-regulation of inflammation-related LncRNA-IL7R predicts poor clinical outcome in patients with cervical cancer. Biosci Rep 38(3):BSR20180483

Fardilha M, Esteves SL, Korrodi-Gregório L et al (2011) Identification of the human testis protein phosphatase 1 interactome. Biochem Pharmacol 82(10):1403-1415

Fu Y, Liu S, Zeng S et al (2019) From bench to bed: the tumor immune microenvironment and current immunotherapeutic strategies for hepatocellular carcinoma. J Exp Clin Cancer Res 38(1):396

Galle PR, Tovoli F, Foerster F et al (2017) The treatment of intermediate stage tumours beyond TACE: from surgery to systemic therapy. J Hepatol 67(1):173-183

Germain C, Meier A, Jensen T et al (2011) Induction of lectin-like transcript 1 (LLT1) protein cell surface expression by pathogens and interferon- $\gamma$ contributes to modulate immune responses. $\mathrm{J}$ Biol Chem 286(44):37964-37975

Giannini EG, Farinati F, Ciccarese F et al (2015) Prognosis of untreated hepatocellular carcinoma. Hepatology 61(1):184-190

Hanahan D, Weinberg RA (2011) Hallmarks of cancer: the next generation. Cell 144(5):646-674

Hato T, Goyal L, Greten TF et al (2014) Immune checkpoint blockade in hepatocellular carcinoma: current progress and future directions. Hepatology 60(5):1776-1782

He G, Fu S, Li Y et al (2020a) TCGA and ESTIMATE data mining to identify potential prognostic biomarkers in HCC patients. Aging (Albany NY) 12(21):21544-21558

He K, Liu S, Xia Y et al (2020b) CXCL12 and IL7R as novel therapeutic targets for liver hepatocellular carcinoma are correlated with somatic mutations and the tumor immunological microenvironment. Front Oncol 10:574853

Hedl M, Zheng S, Abraham C (2014) The IL18RAP region disease polymorphism decreases IL18RAP/IL-18R1/IL-1R1 expression and signaling through innate receptor-initiated pathways. J Immunol 192(12):5924-5932

Jemal A, Ward EM, Johnson CJ et al (2017) Annual report to the nation on the status of cancer, 1975-2014, featuring survival. J Natl Cancer Inst 109(9):djx030

Jiang Y, Que W, Zhu P et al (2020) The role of diverse liver cells in liver transplantation tolerance. Front Immunol 11:1203

Kapas S, Catt KJ, Clark AJ (1995) Cloning and expression of cDNA encoding a rat adrenomedullin receptor. J Biol Chem 270(43):25344-25347

Kaplanski G (2018) Interleukin-18: biological properties and role in disease pathogenesis. Immunol Rev 281(1):138-153 
Kimura K, Wakamatsu A, Suzuki Y et al (2006) Diversification of transcriptional modulation: largescale identification and characterization of putative alternative promoters of human genes. Genome Res 16(1):55-65

Kudo M (2019) Combination cancer immunotherapy with molecular targeted agents/anti-CTLA-4 antibody for hepatocellular carcinoma. Liver Cancer 8(1):1-11

Kwon HB, Mackie DI, Bonnavion R et al (2020) The orphan G-protein coupled receptor 182 is a negative regulator of definitive hematopoiesis through leukotriene B4 signaling. ACS Pharmacol Transl Sci 3(4):676-689

Lanier LL, Chang C, Phillips JH (1994) Human NKR-P1A. A disulfide-linked homodimer of the C-type lectin superfamily expressed by a subset of $\mathrm{NK}$ and $\mathrm{T}$ lymphocytes. J Immunol 153(6):2417-2428

Le Mercier A, Bonnavion R, Yu W et al (2021) GPR182 is an endothelium-specific atypical chemokine receptor that maintains hematopoietic stem cell homeostasis. Proc Natl Acad Sci USA 118(17):e2021596118

Liang J, Zhao H, Hu J et al (2018) SPOCD1 promotes cell proliferation and inhibits cell apoptosis in human osteosarcoma. Mol Med Rep 17(2):3218-3225

Lin GW, Xu C, Chen K et al (2020) Genetic risk of extranodal natural killer T-cell lymphoma: a genomewide association study in multiple populations. Lancet Oncol 21(2):306-316

Liu D, Yang Y, Yan A et al (2020) SPOCD1 accelerates ovarian cancer progression and inhibits cell apoptosis via the PI3K/AKT pathway. Onco Targets Ther 13:351-359

Llovet JM, Ricci S, Mazzaferro V et al (2008) Sorafenib in advanced hepatocellular carcinoma. N Engl J Med 359(4):378-390

Maggi L, Santarlasci V, Capone M et al (2010) CD161 is a marker of all human IL-17-producing T-cell subsets and is induced by RORC. Eur J Immunol 40(8):2174-2181

Mesci A, Ljutic B, Makrigiannis AP et al (2006) NKR-P1 biology: from prototype to missing self. Immunol Res 35(1-2):13-26

Newman AM, Liu CL, Green MR et al (2015) Robust enumeration of cell subsets from tissue expression profiles. Nat Methods 12(5):453-457

Newman AM, Steen CB, Liu CL et al (2019) Determining cell type abundance and expression from bulk tissues with digital cytometry. Nat Biotechnol 37(7):773-782

Poggi A, Costa P, Morelli L et al (1996) Expression of human NKRP1A by CD34+ immature thymocytes: NKRP1A-mediated regulation of proliferation and cytolytic activity. Eur J Immunol 26(6):1266-1272

Poggi A, Costa P, Tomasello E et al (1998) IL-12-induced up-regulation of NKRP1A expression in human NK cells and consequent NKRP1A-mediated down-regulation of NK cell activation. Eur J Immunol 28(5):1611-1616

Rosen DB, Cao W, Avery DT et al (2008) Functional consequences of interactions between human NKR-P1A and its ligand LLT1 expressed on activated dendritic cells and B cells. J Immunol 180(10):6508-6517

Shlomai A, de Jong YP, Rice CM (2014) Virus associated malignancies: the role of viral hepatitis in hepatocellular carcinoma. Semin Cancer Biol 26:78-88

Sung H, Ferlay J, Siegel RL et al (2021) Global cancer statistics 2020: GLOBOCAN estimates of incidence and mortality worldwide for 36 cancers in 185 countries. CA Cancer J Clin 71(3):209-249

Tumeh PC, Hellmann MD, Hamid O et al (2017) Liver metastasis and treatment outcome with anti-PD-1 monoclonal antibody in patients with melanoma and NSCLC. Cancer Immunol Res 5(5):417-424

Turtle CJ, Delrow J, Joslyn RC et al (2011) Innate signals overcome acquired TCR signaling pathway regulation and govern the fate of human CD161(hi) CD8 $\alpha^{+}$semi-invariant $\mathrm{T}$ cells. Blood 118(10):2752-2762

Unitt E, Marshall A, Gelson W et al (2006) Tumour lymphocytic infiltrate and recurrence of hepatocellular carcinoma following liver transplantation. J Hepatol 45(2):246-253

van der Heijden AG, Mengual L, Lozano JJ et al (2016) A five-gene expression signature to predict progression in T1G3 bladder cancer. Eur J Cancer 64:127-136

Wang YM, Li ZX, Tang FB et al (2016) Association of genetic polymorphisms of interleukins with gastric cancer and precancerous gastric lesions in a high-risk Chinese population. Tumour Biol 37(2):2233-2242

Weinmann A, Galle PR (2020) Role of immunotherapy in the management of hepatocellular carcinoma: current standards and future directions. Curr Oncol 27(13):152-164 
Woller N, Gürlevik E, Fleischmann-Mundt B et al (2015) Viral infection of tumors overcomes resistance to PD-1-immunotherapy by broadening neoantigenome-directed T-cell responses. Mol Ther 23(10):1630-1640

Xiang S, Li J, Shen J et al (2021) Identification of prognostic genes in the tumor microenvironment of hepatocellular carcinoma. Front Immunol 12:653836

Xiao L, Harrell JC, Perou CM et al (2014) Identification of a stable molecular signature in mammary tumor endothelial cells that persists in vitro. Angiogenesis 17(3):511-518

Yin L, Zhou L, Xu R (2020) Identification of tumor mutation burden and immune infiltrates in hepatocellular carcinoma based on multi-omics analysis. Front Mol Biosci 7:599142

Yoshihara K, Shahmoradgoli M, Martínez E et al (2013) Inferring tumour purity and stromal and immune cell admixture from expression data. Nat Commun 4:2612

Yu S, Cai L, Liu C et al (2021) Identification of prognostic alternative splicing events related to the immune microenvironment of hepatocellular carcinoma. Mol Med 27(1):36

Zhang HH, Mei MH, Fei R et al (2010) Regulatory T cells in chronic hepatitis B patients affect the immunopathogenesis of hepatocellular carcinoma by suppressing the anti-tumour immune responses. J Viral Hepat 17(Suppl 1):34-43

Zhang G, Liu Y, Dong F et al (2020) Transcription/expression of KLRB1 gene as a prognostic indicator in human esophageal squamous cell carcinoma. Comb Chem High Throughput Screen 23(7):667-674

Zhernakova A, Festen EM, Franke L et al (2008) Genetic analysis of innate immunity in Crohn's disease and ulcerative colitis identifies two susceptibility loci harboring CARD9 and IL18RAP. Am J Hum Genet 82(5):1202-1210

Zhu M, Yan C, Ren C et al (2017) Exome array analysis identifies variants in SPOCD1 and BTN3A2 that affect risk for gastric cancer. Gastroenterology 152(8):2011-2021

Zimmermann FA, Davies HS, Knoll PP et al (1984) Orthotopic liver allografts in the rat. The influence of strain combination on the fate of the graft. Transplantation 37(4):406-410

Publisher's Note Springer Nature remains neutral with regard to jurisdictional claims in published maps and institutional affiliations.

\section{Authors and Affiliations}

\section{Zijun Zhao ${ }^{1} \cdot$ Chaonan Wang $^{2} \cdot$ Peishan $\mathrm{Chu}^{3} \cdot \mathrm{Xin} \mathrm{Lu}^{1}$ (D)}

1 Department of Liver Surgery, Peking Union Medical College Hospital, Chinese Academy of Medical Sciences and Peking Union Medical College, 1 Shuaifuyuan, Wangfujing, Beijing 100730, China

2 Department of Vascular Surgery, Peking Union Medical College Hospital, Chinese Academy of Medical Sciences and Peking Union Medical College, Beijing, China

3 Department of Cardiac Surgery, Peking Union Medical College Hospital, Chinese Academy of Medical Sciences and Peking Union Medical College, Beijing, China 